\title{
CLOSED GEODESICS ON PAIRS OF PANTS
}

\author{
BY \\ Clatre M. Baribaud* \\ Département de Mathématiques, Ecole Polytechnique Fédérale de Lausanne \\ CH-1015 Lausanne, Switzerland \\ e-mail: baribaud@dma.epfl.ch
}

\begin{abstract}
We study the non-simple closed geodesics of the Riemann surfaces of signature $(0,3)$. In the aim of classifying them, we define one parameter: the number of strings. We show that for a given number of strings, a minimal geodesic exists; we then give its representation and its length which depends on the boundary geodesics.
\end{abstract}

\section{Introduction}

A Riemann surface of signature $(g, n)$ is an oriented, connected surface of genus $g$ with $n$ boundary components, called boundary geodesics, which is equipped with a metric of constant curvature -1 . The length spectrum is the set, listed in ascending order, of lengths of closed geodesics of a Riemann surface [Hub59]. Almost every compact Riemann surface (i.e. a surface without boundary components) is determined by its length spectrum, up to isometry [Bus92]. Such surfaces can be decomposed into a succession of Riemann surfaces of signature $(0,3)$ more commonly called a pair of pants, so it is important to study the length spectrum of a pair of pants. Moreover, the first elements of the length spectrum of a surface yield a lot of information about the surface itself, thus we are interested in the study of geodesics having a short length. One of the main difficulties is to "catch" every geodesic below a given length. An easy topological criterion, which would permit us to group on one hand short geodesics and on the other hand long geodesics, is still missing. In this article, we define a parameter - the number of strings - and we classify geodesics using this parameter.

* Current address: Department of Mathematics, ETH-Zentrum, 8029 Zürich, Switzerland.

Received November 6, 1997 


\section{Definitions and preliminaries}

Let $Y$ be a Riemann surface of signature $(0,3)$ and $\gamma_{1}, \gamma_{2}, \gamma_{3}$ its boundary geodesics. Let us denote by $p_{1}, p_{2}, p_{3}$ the perpendicular segments which respectively link $\gamma_{2}$ to $\gamma_{3}, \gamma_{1}$ to $\gamma_{3}$ and $\gamma_{1}$ to $\gamma_{2}$. The boundary geodesics are numbered such that $0<\ell\left(\gamma_{1}\right) \leqslant \ell\left(\gamma_{2}\right) \leqslant \ell\left(\gamma_{3}\right)$, where $\ell\left(\gamma_{i}\right)$ is the hyperbolic length of the geodesic $\gamma_{i}, i=1,2,3$. To simplify notation, we will write $\gamma$ instead of $\ell(\gamma)$ for any geodesic $\gamma$ (and by "geodesic" we refer to the shortest curve in the considered homotopy class). The Riemann surface $Y$ can be split into two geodesic hexagons $Y_{1}$ and $Y_{2}$. Let $\sigma$ be the symmetry which maps $Y_{1}$ onto $Y_{2}\left(\sigma\left(p_{i}\right)=p_{i}\right.$, $i=1,2,3)$.

Definition 2.1: A string is a segment of curve which links two perpendiculars $p_{i}, p_{j}, i, j=1,2,3, i \neq j$ and has no other intersection with $p_{1}, p_{2}, p_{3}$.

A closed geodesic is made of a succession of $2 n$ strings $t_{1}, \ldots, t_{2 n}, n \in \mathbf{N}^{*}$, such that two consecutive strings do not belong to the same $Y_{i}, i=1,2$. We remark also that a closed geodesic always has an even number of strings.

Definition 2.2: Let $g$ be a closed geodesic with $2 n$ strings. $g$ is said to be zygomorphic if it is made of a succession of strings $t_{1}, \ldots, t_{2 n}$ such that $\sigma\left(t_{k}\right)=$ $t_{2 n+1-k}$, where $1 \leqslant k \leqslant n$. We call such a succession a good succession.

Notice that a good succession can be decomposed into two half-paths $d_{1}=$ $t_{1}, \ldots, t_{n}, d_{2}=t_{n+1}, \ldots, t_{2 n}$ such that $\sigma\left(d_{1}\right)=d_{2}$.

Finally, we adopt the following notation:

$$
\begin{aligned}
c_{i} & =\cosh \left(\frac{1}{2} \gamma_{i}\right), \quad c_{i, n}=\cosh \left(n \frac{1}{2} \gamma_{i}\right), \\
s_{i}=\sinh \left(\frac{1}{2} \gamma_{i}\right), & s_{i, n}=\sinh \left(n \frac{1}{2} \gamma_{i}\right), \quad i=1,2,3 .
\end{aligned}
$$

\section{Results}

We call $g_{n, m}^{i j}$ the zygomorphic closed geodesic having one of its good halfsuccessions $d_{q}, q=1,2$ made of $n$ strings on the $\gamma_{i}$-leg, and $m$ strings on the $\gamma_{j}-\operatorname{leg}, i, j=1,2,3, i \neq j$ and $n, m \in \mathbf{N}^{*}$ (cf. Figure 1). 


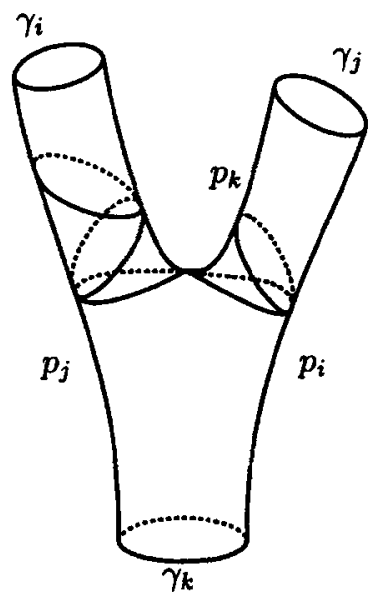

Figure 1. Geodesic $g_{3,2}^{i j}$.

The lift of $d_{q}$ in the hyperbolic plane $\mathbf{H}$ is an edge of a crossed right-angled hexagon. So we obtain the explicit length of $g_{n, m}^{i j}$ given by the following relation,

$$
\cosh \left(\frac{1}{2} g_{n, m}^{i j}\right)=\frac{s_{i, n} s_{j, m}}{s_{i} s_{j}}\left(c_{k}+c_{i} c_{j}\right)+c_{i, n} c_{j, m}, \quad 1 \leqslant i \leqslant j \leqslant 3
$$

From now on we will write $G_{n, m}^{i j}:=\cosh \left(\frac{1}{2} g_{n, m}^{i j}\right)$.

We now state our main result in the following theorem.

THEOREM 3.1: $g_{n, 1}^{12}$ is the shortest closed geodesic having at least $2(n+1)$ strings.

\section{Proof of Theorem 3.1}

We begin by classifying the $g^{i j}$-geodesics described above according to their number of strings. To prove the two propositions below, we need to use just basic properties of the hyperbolic functions.

Proposition 4.1: If $n \geqslant m$, then $g_{n m}^{i j} \leqslant g_{m n}^{i j}, 1 \leqslant i<j \leqslant 3$.

Proof: We are going to prove that

$$
G_{n m}^{i j}-G_{m n}^{i j} \leqslant 0
$$

By expanding the above expression, we have

$$
\frac{c_{k}+c_{i} c_{j}}{s_{i} s_{j}}\left(s_{i, n} s_{j, m}-s_{i, m} s_{j, n}\right)+\left(c_{i, n} c_{j, m}-c_{i, m} c_{j, n}\right) \leqslant 0 .
$$


As $n \geqslant m$, the functions

$$
x \longmapsto \frac{\sinh (n x)}{\sinh (m x)} \quad \text { and } \quad x \longmapsto \frac{\cosh (n x)}{\cosh (m x)}
$$

are increasing. Moreover, $\left(c_{k}+c_{i} c_{j}\right) / s_{i} s_{j}>0$.

Proposition 4.2: For all $n, m \in \mathbf{N}^{*}$, we have

$$
g_{n, m}^{12} \leqslant g_{n, m}^{13} \leqslant g_{n, m}^{23} .
$$

Proof: By definition $0<\gamma_{1} \leqslant \gamma_{2} \leqslant \gamma_{3}$. This implies that

$$
0<c_{3}+c_{1} c_{2} \leqslant c_{2}+c_{1} c_{3} \leqslant c_{1}+c_{1} c_{3} .
$$

Moreover, $\sinh (k x) / \sinh (x)$ is an increasing function of $x \in \mathbf{R}_{+}\left(k \in \mathbf{N}^{*}\right)$.

In order to prove the next proposition, we use the Chebyshev polynomials of the second kind:

$$
U_{n}(x)=\frac{\sin (n \arccos (x))}{\sin (\arccos (x))}
$$

These polynomials verify the relation $U_{n+1}(x)=2 x U_{n}(x)-U_{n-1}(x)$ and so does

$$
G_{n, m}^{i j}=2 c_{i} G_{n-1, m}^{i j}-G_{n-2, m}^{i j}=2 c_{j} G_{n, m-1}^{i j}-G_{n, m-2}^{i j}
$$

from which we deduce the useful relations:

$$
\begin{array}{ll}
G_{n, m}^{i j}=U_{n-1}\left(c_{i}\right) G_{2, m}^{i j}-U_{n-2}\left(c_{i}\right) G_{1, m}^{i j}, & \forall n \leqslant 2 \text { and } \forall m \leqslant 1, \\
G_{n, m}^{i j}=U_{m-1}\left(c_{j}\right) G_{n, 2}^{i j}-U_{m-2}\left(c_{j}\right) G_{n, 1}^{i j}, & \forall n \leqslant 1 \text { and } \forall m \leqslant 2,
\end{array}
$$

which are easy to prove by induction.

Proposition 4.3: For all $n, m \in \mathbf{N}^{*}$ such that $n>m$ and $1 \leqslant i<j \leqslant 3$, we have

$$
g_{n, m}^{i j} \leqslant g_{n-1, m+1}^{i j} .
$$

Proof: Using $(*)$, we can write

$$
G_{n, m}^{i j}-G_{n-1, m+1}^{i j}=2\left(c_{i}-c_{j}\right) G_{n-1, m}^{i j}+G_{n-1, m-1}^{i j}-G_{n-2, m}^{i j} .
$$

The proposition is equivalent to showing that $G_{n, m}^{i j}-G_{n-1, m+1}^{i j} \leqslant 0$. This relation is true only if $G_{n-1, m-1}^{i j}-G_{n-2, m}^{i j} \leqslant 0$. Because $n>m$, using induction, we reduce the problem to the study of the sign of the following expression,

$$
G_{l, 1}^{i j}-G_{l-1,2}^{i j}, \quad \text { where } l \geqslant 2 .
$$


If $l=2$, then $G_{2,1}^{i j}-G_{1,2}^{i j}=\left(4 c_{i} c_{j}+2 c_{k}+1\right)\left(c_{i}-c_{j}\right) \leqslant 0$.

If $l=3$, then $G_{3,1}^{i j}-G_{2,2}^{i j}=\left(8 c_{i} c_{j}^{2}-2 c_{i}-2 c_{j}+4 c_{i} c_{j}\right)\left(c_{i}-c_{j}\right)-1-c_{k} \leqslant 0$.

If $l \geqslant 3$, then $G_{l, 1}^{i j}-G_{l-1,2}^{i j}=U_{l-2}\left(c_{i}\right)\left(G_{3,1}^{i j}-G_{2,2}^{i j}\right)+U_{l-3}\left(c_{i}\right)\left(G_{1,2}^{i j}-G_{2,1}^{i j}\right)$.

We conclude by remarking that $8 c_{i} c_{j}^{2}-2 c_{i}-2 c_{j}+4 c_{i} c_{j} \geqslant 4 c_{i} c_{j}+2 c_{k}$ and $c_{k}+1 \geqslant c_{j}-c_{i}$.

Propositions 4.1-4.3 together imply that $g_{n-1,1}^{12}$ is the shortest geodesic among the geodesics $g_{r, s}^{i j}$ where $r+s=n(i<j)$ (thus proving a particular case of Theorem 3.1).

We call $h_{n m}^{i j}$ the zygomorphic geodesic having one of its good half-successions $d_{q}, q=1,2$ made of $n$ strings on the $\gamma_{i}$-leg, one string on the $\gamma_{k}$-leg and $m$ strings on the $\gamma_{j}$-leg, $i, j, k=1,2,3, i \neq j$ and $n, m \in \mathbf{N}^{*}$ (cf. Figure 2).

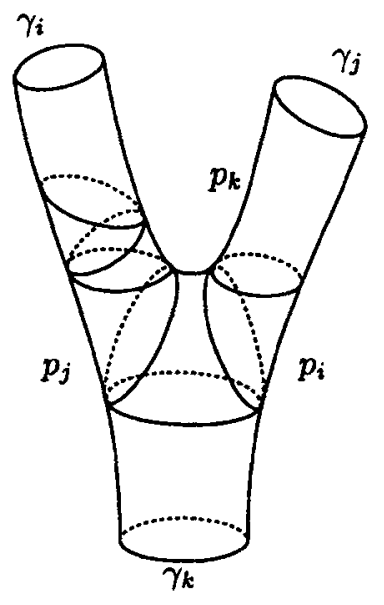

Figure 2. Geodesic $h_{4,2}^{i j}$.

The lift of $d_{q}$ in the hyperbolic plane $\mathbf{H}$ is an edge of a right-angled hexagon. So we obtain the explicit length of $h_{n, m}^{i j}$ given by the following relation,

$$
\cosh \left(\frac{1}{2} h_{n, m}^{i j}\right)=\frac{s_{i, n+1} s_{j, m+1}}{s_{i} s_{j}}\left(c_{k}+c_{i} c_{j}\right)-c_{i, n+1} c_{j, m+1}, \quad 1 \leqslant i \leqslant j \leqslant 3 .
$$

From now on we will write $H_{n, m}^{i j}:=\cosh \left(\frac{1}{2} h_{n, m}^{i j}\right)$.

Now we compare these two different types of zygomorphic geodesics.

Proposition 4.4: For all $n, m \in \mathbf{N}^{*}$, we have

$$
g_{n+1, m}^{i j} \leqslant h_{n, m}^{i j}, \quad 1 \leqslant i<j \leqslant 3 .
$$


Proof: In fact, we are going to prove $G_{n+1, m}^{i j} \leqslant H_{n, m}^{i j}$. Let $e_{k}=c_{k}+c_{i} c_{j}$. We split $H_{n, m}^{i j}$ such that it is easy to compare it with $G_{n+1, m}^{i j}$. Let us write

$$
H_{n m}^{i j}=h_{1}+h_{2}+h_{3}+h_{4} \quad \text { and } \quad G_{n+1, m}^{i j}=g_{1}+g_{2}
$$

where

$$
\begin{array}{cc}
h_{1}=\frac{s_{i, n+1} s_{j, m}}{s_{i} s_{j}} c_{j} e_{k}, & h_{3}=c_{i, n+1} c_{j, m} c_{j}, \\
h_{2}=\frac{s_{i, n+1} c_{j, m}}{s_{i}} e_{k}, & h_{1}=c_{i, n+1} s_{j, m} s_{j}, \\
g_{1}=\frac{s_{i, n+1} s_{j, m}}{s_{i} s_{j}} e_{k}, & g_{2}=c_{i, n+1} c_{j, m} .
\end{array}
$$

As $h_{1}=g_{1}+g_{1}\left(c_{j}-1\right)$, we denote $h_{1}^{\prime}=g_{1}\left(c_{j}-1\right)$. Now it is easy to show that

$$
g_{2}+h_{3} \leqslant h_{2} \quad \text { and } \quad h_{4} \leqslant h_{1}^{\prime} .
$$

Proposition 4.5: Let $g$ be a zygomorphic closed geodesic with $2 n$ strings. Then $g$ can be split into a succession of curves, each one homotopic to a $g_{r, s}^{i j}$ or $h_{p, q^{-}}^{i j}$ geodesic, $1 \leqslant i<j \leqslant 3$ and $r, s, p, q \in \mathrm{N}^{*}$.

Proof: Denote by $d_{1}=t_{1}, \ldots, t_{n}$ the $g$-half-path such that $d_{1} \cup \sigma\left(d_{1}\right)$ is a good $g$-succession.

If $n=2$, it is easy to see that $g=g_{1,1}^{i j}, i, j=1,2,3$ distinct.

If $n=3$, then $g=g_{2,1}^{i j}$ or $g=h_{1,1}^{i j}, i, j=1,2,3$ distinct.

Let $n>3$ and suppose that the good succession above can be split into a good succession of curves $c_{1}, \ldots, c_{r}$, each one homotopic to a geodesic $g_{r, s}^{i j}$ or $h_{p, q}^{i j}$, $r, s, p, q \in \mathbf{N}^{*}$, called respectively $g_{1}, \ldots, g_{r}$. Let $d_{2}=d_{1} \cup t_{n+1}$. Then $d_{2} \cup \sigma\left(d_{2}\right)$ is homotopic to a geodesic $g^{\prime}$ having $2(n+1)$ strings. Let us show that $g^{\prime}$ can be also split into a succession as described above. Let us suppose that $g_{r}=g_{u, v}^{i j}$ and denote by $d_{r}=t_{n+1-(u+v)}, \ldots, t_{n}$ the path included in $d_{1}$ such that $d_{r} \cup \sigma\left(d_{r}\right)$ is homotopic to $g_{r}$ and $t_{n}$ is on the $\gamma_{j}$-leg. Let $d_{2}^{\prime}=d_{r} \cup t_{n+1}$.

1. If $t_{n+1}$ is also located on the $\gamma_{j}$ leg, then $d_{2}^{\prime} \cup \sigma\left(d_{2}^{\prime}\right)$ is homotopic to $g_{r}=$ $g_{u, v+1}^{i j}$.

2. If $t_{n+1}$ is located on the $\gamma_{q}$-leg, $q \neq j$,

a. if $v=1$, then $d_{2}^{\prime} \cup \sigma\left(d_{2}^{\prime}\right)$ is homotopic to $g_{r}=h_{u, 1}^{i k}$;

b. if $v>1$, denote $f=t_{n+1-(u+v)}, \ldots, t_{n-1}$; then $f \cup \sigma(f)$ is homotopic to $g_{r}=g_{u, v-1}^{i j}$ and $\left(t_{n} \cup t_{n+1}\right) \cup \sigma\left(t_{n} \cup t_{n+1}\right)$ is homotopic to the new element $g_{r+1}=g_{1,1}^{j, q}$.

If $g_{r}=h_{s, t}^{i j}$, the proof is similar to the above one. 
Proposition 4.6: For all $1 \leqslant m \leqslant n-2$ and $1 \leqslant i<j \leqslant 3$,

$$
g_{n, 1}^{i j} \leqslant g_{n-m-1,1}^{i j}+g_{m, 1}^{i j} .
$$

Proof: We reduce the problem to the following inequality,

$$
G_{n, 1}^{i j} \leqslant G_{n-m-1,1}^{i j} G_{m, 1}^{i j}+\sqrt{\left.\left(G_{n-m-1,1}^{i j}\right)^{2}-1\right)} \sqrt{\left(G_{m, 1}^{i j}\right)^{2}-1}
$$

and we split the problem into two parts.

Firstly, we show that

$$
\frac{s_{i, n}}{s_{i}}\left(c_{k}+c_{i} c_{j}\right) \leqslant G_{n-m-1,1}^{i j} G_{m, 1}^{i j} \cdot c_{i, n}^{2} c_{j}^{2} \leqslant \sqrt{\left.\left(G_{n-m-1,1}^{i j}\right)^{2}-1\right)} \sqrt{\left(G_{m, 1}^{i j}\right)^{2}-1} .
$$

Let again $e_{k}=c_{k}+c_{i} c_{j}$. Let us write

$$
h_{1}=\frac{s_{i, n}}{s_{i}}\left(c_{k}+c_{i} c_{j}\right) \quad \text { and } \quad h_{2}=G_{n-m-1,1}^{i j} G_{m, 1}^{i j} .
$$

Now we split $h_{1}$ and $h_{2}$ such that $h_{1}=h_{11}+\cdots+h_{15}$ and $h_{2}=h_{21}+\cdots+h_{25}$ where

$$
\begin{array}{ll}
h_{11}=\frac{s_{i, n-m-1}}{s_{i}} c_{i, m} c_{i} e_{k}, & h_{12}=s_{i, n-m-1} s_{i, m} e_{k}, \\
h_{13}=\frac{c_{i, n-m-1}}{s_{i}} s_{i, m} c_{i} e_{k}, & h_{14}=c_{i, n-m-1} c_{i, m} c_{i} c_{j} \\
h_{15} & =c_{i, n-m-1} c_{i, m} c_{k},
\end{array}
$$

and

$$
\begin{array}{ll}
h_{21}=\frac{s_{i, n-m-1}}{s_{i}} c_{i, m} c_{j} e_{k}, & h_{22}=\frac{s_{i, n-m-1}}{s_{i}^{2}} s_{i, m}\left(c_{k}^{2}+c_{i}^{2} c_{j}^{2}\right), \\
h_{23}=c_{i, n-m-1} c_{j} \frac{s_{i, m}}{s_{i}} e_{k}, & h_{24}=c_{i, n-m-1} c_{i, m} c_{j}^{2}, \\
h_{25}=2 \frac{s_{i, n-m-1}}{s_{i}^{2}} s_{i, m} c_{i} c_{j} c_{k} . &
\end{array}
$$

It is easy to see that $h_{1 i} \leqslant h_{2 i}, i=1, \ldots, 5$.

Secondly, we have to prove

$$
c_{i, n}^{2} c_{j}^{2} \leqslant\left(\left(G_{n-m-1,1}^{i j}\right)^{2}-1\right)\left(\left(G_{m, 1}^{i j}\right)^{2}-1\right) .
$$

In order to simplify the notation, let us set $f_{k}^{2}=2 c_{i} c_{j} c_{k}+c_{i}^{2} c_{j}^{2} \geqslant 3$. We have the following inequality,

$$
\frac{s_{i, r}^{2}}{s_{i}^{2}} e_{k}^{2}-1 \geqslant \frac{s_{i, r}^{2}}{s_{i}^{2}} f_{k}^{2}
$$


Let us write

$$
\begin{aligned}
k_{1}= & c_{i, n}^{2} c_{j}^{2} \\
k_{2}= & \left(\frac{s_{i, n-m-1}^{2}}{s_{i}^{2}} f_{k}^{2}+2 \frac{s_{i, n-m-1}}{s_{i}} c_{i, n-m-1} c_{j} e_{k}+c_{i, n-m-1}^{2} c_{j}^{2}\right) \\
& \times\left(\frac{s_{i, m}^{2}}{s_{i}^{2}} f_{k}^{2}+2 \frac{s_{i, m}}{s_{i}} c_{i, m} c_{j} e_{k}+c_{i, m}^{2} c_{j}^{2}\right) .
\end{aligned}
$$

We are going to prove that $k_{1} \leqslant k_{2}$. We split $k_{1}$ and $k_{2}$ such that

$$
k_{1}=k_{11}+\cdots+k_{19} \quad \text { and } \quad k_{2}=k_{21}+\cdots+k_{29}
$$

where

$$
\begin{aligned}
& k_{11}=c_{i, n-m-1}^{2} c_{i, m}^{2} c_{i}^{2} c_{j}^{2}, \quad k_{12}=c_{i, n-m-1}^{2} s_{i, m}^{2} s_{i}^{2} c_{j}^{2}, \\
& k_{13}=s_{i, n-m-1}^{2} s_{i, m}^{2} c_{i}^{2} c_{j}^{2}, \quad k_{14}=s_{i, n-m-1}^{2} c_{i, m}^{2} s_{i}^{2} c_{j}^{2}, \\
& k_{15}=2 c_{i, n-m-1}^{2} c_{i, m} s_{i, m} c_{i} s_{i} c_{j}^{2}, \\
& k_{16}=2 s_{i, n-m-1}^{2} c_{i, m} s_{i, m} c_{i} s_{i} c_{j}^{2}, \\
& k_{17}=2 c_{i, n-m-1} s_{i, n-m-1} c_{i, m} s_{i, m}\left(s_{i}^{2}+c_{i}^{2}\right) c_{j}^{2}, \\
& k_{18}=2 c_{i, n-m-1} s_{i, n-m-1} c_{i} s_{i} s_{i, m}^{2} c_{j}^{2}, \\
& k_{19}=2 c_{i, n-m-1} s_{i, n-m-1} c_{i} s_{i} c_{i, m}^{2} c_{j}^{2},
\end{aligned}
$$

and

$$
\begin{aligned}
& k_{21}=c_{i, n-m-1}^{2} c_{i, m}^{2} c_{j}^{2} c_{j}^{2}, \quad k_{22}=c_{i, n-m-1}^{2} c_{j}^{2} \frac{s_{i, m}^{2}}{s_{i}^{2}} f_{k}^{2}, \\
& k_{23}=\frac{s_{i, n-m-1}^{2}}{s_{i}^{2}} f_{k}^{2} \frac{s_{i, m}^{2}}{s_{i}^{2}} f_{k}^{2}, \quad k_{24}=\frac{s_{i, n-m-1}^{2}}{s_{i}^{2}} f_{k}^{2} c_{i, m}^{2} c_{j}^{2} \\
& k_{25}=2 c_{i, n-m-1}^{2} c_{j}^{2} \frac{s_{i, m}}{s_{i}} e_{k} c_{i, m} c_{j}, \\
& k_{26}=2 \frac{s_{i, n-m-1}^{2}}{s_{i}^{2}} f_{k}^{2} \frac{s_{i, m}}{s_{i}} e_{k} c_{i, m} c_{j}, \\
& k_{27}=4 \frac{s_{i, n-m-1}}{s_{i}} e_{k} c_{i, n-m-1} c_{j} \frac{s_{i, m}}{s_{i}} e_{k} c_{i, m} c_{j}, \\
& k_{28}=2 c_{i, n-m-1} c_{j} \frac{s_{i, n-m-1}}{s_{i}} e_{k} c_{i, m}^{2} c_{j}^{2}, \\
& k_{29}=2 c_{i, n-m-1} c_{j} \frac{s_{i, n-m-1}}{s_{i}} e_{k} \frac{s_{i, m}^{2}}{s_{i}^{2}} f_{k}^{2} .
\end{aligned}
$$

We see that $k_{1 i} \leqslant k_{2 i}, i=1, \ldots, 9$, hence the result. 
Proposition 4.7: Let $g$ be a non-zygomorphic closed geodesic with $2 n$ strings. Then there is at least one zygomorphic closed geodesic with $2 n$ strings which is shorter than $g$.

Proof: The geodesic $g$ has an even number of strings, so we can split it into two paths $c_{1}$ and $c_{2}$ which are not homotopic to a boundary geodesic and have $n$ strings each. Denote by $g_{i}$ the (non-closed) geodesic which is homotopic to $c_{i}$, $i=1,2$. Assume that $g_{1}$ is the shortest path. Then $g_{1} \cup \sigma\left(g_{1}\right)$ is a zygomorphic geodesic with $2 n$ strings which is shorter than $g$.

So we can assert that $g_{n, 1}^{12}$ is the shortest closed geodesic having $2(n+1)$ strings. Moreover, $g_{n, 1}^{12} \leqslant g_{n+1,1}^{12}, n \in \mathbf{N}^{*}$; this means also that $g_{n, 1}^{12}$ is the shortest closed geodesic having at least $2(n+1)$ strings as claimed, and ends the proof of Theorem 3.1 .

Remark 4.8: The minimum of $G_{n, 1}^{12}$ is equal to $2 n+1$.

$G_{n, 1}^{12}$ is minimal when the boundary geodesics are cusps. This special case of a pair of pants was studied by Paul Schmutz Schaller [Sch96b] who gave its length spectrum without counting multiplicity, using a very different approach than the one taken here. The length spectrum is $\{2 n+1 \mid n \in \mathbf{N}\}$. He gave also the length spectrum of a special pairs of pants with two cusps in [Sch96a].

\section{References}

[Bus92] P. Buser, Geometry and spectra of compact Riemann surfaces, in Progress in Mathematics, Vol. 106, Birkhäuser, Boston, Basel, Berlin, 1992.

[Hub59] H. Huber, Zur analytischen Theorie hyperbolischer Raumformen und Bewegungsgruppen I, Mathematische Annalen 138 (1959), 1-26.

[Sch96a] P. Schmutz Schaller, Arithmetic groups and the length spectrum of riemann surfaces, Duke Mathematical Journal 84 (1996), 199-215.

[Sch96b] P. Schmutz Schaller, The modular torus has maximal length spectrum, Geometric and Functional Analysis 6 (1996), 1057-1073. 